\title{
Gut microbiota produce alcohol in patients with NASH
}

NASH has a poor prognosis. It currently accounts for $10 \%$ of liver transplantations conducted in the USA and is predicted to soon become the most common indication for liver transplantation. "Understanding the pathophysiology of NASH is therefore of crucial importance," explains Lixin Zhu, corresponding author of a study published in Hepatology. "Histologically, NASH is indistinguishable from alcoholic liver disease, so we predicted that alcohol also has a role in NASH."

Previous data from this group revealed that global gene expression in livers from patients with NASH was distinct from that of healthy livers. Interestingly, although dietary alcohol intake was similar, the expression of all genes involved in alcohol metabolism was greater in NASH livers than healthy livers. "We concluded that the only possible source of alcohol in patients with NASH was the gut microbiome," says Zhu.

The aim of this study was simple: identify and quantitate the microbial species in the gut of obese patients with $\mathrm{NASH}$, and compare them with those identified in the gut of obese patients without NASH and healthy individuals as controls. The investigators pyrosequenced $16 \mathrm{~S}$ rRNA extracted from the gut microbiota of these patients and healthy controls. Alcohol concentration in the circulation was also determined.

"Our most striking observation was that the microbiome composition of the patients with NASH was distinct from that of obese patients without NASH and healthy controls," states Zhu. Further analysis revealed that the only major difference between obese patients with NASH and those without NASH was in the genus Escherichia (phylum Proteobacteria). Indeed, the abundance of Escherichia was fivefold higher in obese patients with NASH than those without, revealing an increased abundance of alcohol-producing bacteria in the NASH microbiome.

The serum alcohol concentration was also higher in obese patients with NASH than obese patients without NASH and healthy controls. "This observation is noteworthy as alcohol is capable of inducing liver inflammation and so lends powerful support for a role of alcohol in NASH pathophysiology," says Susan S. Baker, co-corresponding author.

The authors have not, in this study, proven a causal effect between altered microbiota and NASH. Further studies are now needed to address if the course

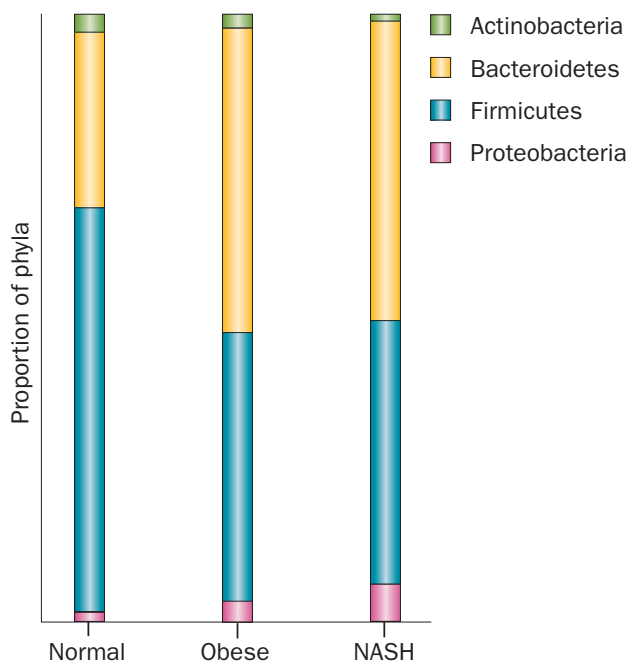

Phylum distribution in gut microbiota of obese patients with and without NASH. Courtesy of L. Zhu.

of NASH can be altered by changing the microbiota of these patients and, if so, what is the optimal approach.

Katherine Smith

Original article Zhu, L. et al. Characterization of the gut microbiome in non-alcoholic steatohepatitis (NASH) patients: a connection between endogenous alcohol and NASH. Hepatology doi:10.1002/hep.26093 